See discussions, stats, and author profiles for this publication at: https://www.researchgate.net/publication/224347219

\title{
Dynamic Output Feedback for Discrete-Time Systems Under Amplitude and Rate Actuator Constraints
}

Article in IEEE Transactions on Automatic Control · December 2008

DOI: 10.1109/TAC.2008.2007521 · Source: IEEE Xplore

CITATIONS

39

4 authors, including:

D. Limon

Universidad de Sevilla

112 PUBLICATIONS 2,508 CITATIONS

SEE PROFILE

(2)

Eduardo F. Camacho

Universidad de Sevilla

389 PUBLICATIONS 12,514 CITATIONS

SEE PROFILE

Some of the authors of this publication are also working on these related projects:

Project HYCON2 View project

Project Economic Model Predictive Control View project
READS

57

Teodoro Alamo
Universidad de Sevilla
240 PUBLICATIONS 4,619 CITATIONS
SEE PROFILE




\title{
Dynamic Output Feedback for Discrete-Time Systems under Amplitude and Rate Actuator Constraints
}

\author{
J.M. Gomes da Silva Jr, D. Limon and T. Alamo
}

\begin{abstract}
The aim of this work is the proposition of a technique for the design of stabilizing dynamic output feedback controllers for discrete-time linear systems with rate and amplitude saturating actuators. The nonlinear effects introduced by the saturations in the closed-loop system are taken into account by using a generalized sector condition, which allows to propose theoretical conditions to solve the problem directly in the form of linear matrix inequalities (LMIs). From these conditions, convex optimization problems to the determination of the controller in order to address the synthesis requirements are proposed. In addition to the asymptotic stability requirement, two implicit design objectives are considered: the maximization of the region of attraction of the closed-loop system and the guarantee of a certain degree of time-domain performance for the system operation in a neighborhood of the origin (equilibrium point). A numerical example is provided to illustrate the application of the proposed method.
\end{abstract}

Keywords: constrained control, control saturation, output feedback, stabilization, discrete-time systems.

\section{INTRODUCTION}

The physical impossibility of applying unlimited control signals makes the actuator saturation an ubiquitous problem in control systems. In particular, it is well known that the input saturation is source of performance degeneration, limit cycles, different equilibrium points, and even instability. Hence, it was great the interest in studying these negative effects and also in proposing control design procedures, in global, semiglobal and local contexts of stability, taking directly into account the control bounds: see for instance [15], [6], [7], and references therein. It should be pointed out that most of these works consider only input amplitude saturation and state feedback control strategies. Although the proposition of state feedback methods allow to have a good insight into the problem, the practical applicability of these methods is limited. On the other hand the works proposing output feedback strategies consider, in general, observer-based control laws ensuring global or semi-global stabilization. However, when the open loop system is not null controllable or additional performance and robustness requirements are needed, local (or regional) stabilization approaches are needed and an implicit additional objective is the enlargement of the basin of attraction of the closed-

J.M Gomes da Silva is with UFRGS - Department of Electrical Engineering. Av. Osvaldo Aranha 103, 90035-190 Porto Alegre-RS, Brazil.

E-mail: jmgomes@ece.ufrgs.br

D. Limon and $\mathrm{T}$. Alamo are with Dept. Ingeniería de Sistemas y Automática. Universidad de Sevilla.

E-mail: \{limon, alamo\}@cartuja.us.es

The authors would like to acknowledge MCYT-Spain (contracts DPI2002 04375-c03-01 and DPI2004-07444-C04-01), CAPES-Brazil (cooperation project 004/2001), CNPq-Brazil (grant 300149/98-0) for funding this work. loop system. In this context, the amount of works proposing output feedback control strategies is even smaller.

Works formally addressing the stabilization in the presence of both amplitude and rate saturation started to appear in the last few years. Global and semi-global stabilization results using both state feedback and observer-based control laws were proposed in [10], [13], [14]. Concerning a local stabilizing context, we can cite the results presented in [4], [1], [16], where the synthesis of state feedback control laws are proposed. On the other hand, the synthesis of dynamic output feedback controllers ensuring local stability is considered in [17] and [11]. In [17], a method for designing dynamic output controllers using of the Positive Real Lemma is proposed. The main objective pursued in that paper is the minimization of an LQG criterion. A region of stability is associated to the closed-loop system. However, it should be pointed out that the size and the shape of this region are not taken into account in the design procedure, which can lead to very conservative domains of stability. Furthermore, the controller is computed from the solution of strong coupled Riccati equations which, in general, are not simple to solve. A time-varying dynamic controller is proposed in [11]. The stabilizing conditions are given in this case in the form of nonlinear matrix inequalities, which implies the use of iterative LMI relaxation schemes for computing the controller. Since the proposed approach considers only continuoustime systems, its main drawback resides in the fact that the stability properties cannot be ensured if the controller is discretized for a digital implementation. Furthermore, in that paper, no explicitly consideration is made about the region of attraction associated to the controller neither about the internal stability of the system. On the other hand, it should be pointed out that all the references above are concerned only with continuous-time systems and the rate limitation is considered in the modeling of the actuator, i.e. a positionfeedback-type model [17] is considered. In this case, the rate saturation is modeled, in fact, as a saturation of the actuator state. Hence, the plant plus the actuator appears as a nonlinear system which renders the formal analysis in the the sampled-data control case quite involved. In this case, an alternative approach consists in designing a digital nonlinear controller (i.e. consider saturations in the controller) in order to prevent that the control signal (to be sent to the actuator) violates both the rate and amplitude bounds.

The aim of this note is the proposition of a technique for the design of stabilizing dynamic output feedback controllers for discrete-time linear systems with rate and amplitude constrained actuators. In addition to the asymptotic stability 
requirement, two implicit design objectives are considered: the maximization of the region of attraction of the closedloop system and the guarantee of a certain degree of timedomain performance for the system operation in a neighborhood of the origin (equilibrium point).

In order to deal with the rate limitation, we propose the synthesis of a nonlinear dynamic controller which is composed by a classical linear dynamic controller in cascade with a input saturating integrator and two static antiwindup loops. It should be pointed out that, differently from the anti-windup approaches (see for instance [8], [5],[3], and references therein), where the controller is supposed to be given, here the idea consists in computing simultaneously the controller and the antiwindup gains. The anti-windup gains appear therefore as extra degrees of freedom in the synthesis problem.

The theoretical conditions for solving the synthesis problem are based on a generalized sector condition proposed in [3]. This condition encompasses the classical sector condition, used for instance in [9], and allows (differently from the classical one) the formulation of local stability conditions directly in LMI form. Using then the classical variables transformations as proposed in [12] and [2], it is possible to formulate conditions that allow to compute a dynamic controller that stabilizes the closed-loop system. Optimization problems to the determination of the controller in order to enlarge the basin of attraction of the closedloop as well as enhance the time-domain performance of the closed-loop system are therefore proposed. A numerical example is provided to illustrate the application of the proposed method.

Notations. $A_{(i)}$ denotes the $i$ th row of matrix $A$. For two symmetric matrices, $A$ and $B, A>B$ means that $A-B$ is positive defnite. $A^{\prime}$ denotes the transpose of $A . \star$ stands for symmetric blocks; $\bullet$ stands for an element that has no induence on the development. $s a t_{\rho}$ is a componentwise saturation map $\Re^{m} \rightarrow \Re^{m}$ de£ned as follows: $\left(\operatorname{sat}_{\rho}(v)\right)_{(i)}=\operatorname{sat}_{\rho_{(i)}}\left(v_{(i)}\right)=\operatorname{sign}\left(v_{(i)}\right) \min \left(\rho_{(i)},\left|v_{(i)}\right|\right) \quad \forall i=$ $1, \ldots, m$, where $\rho_{(i)}$, denotes the $i$ th bound of the saturation function. Given two vectors $x \in \mathfrak{R}^{n}$ and $y \in \mathfrak{R}^{m},(x, y)$ denotes the vector $\left[x^{\prime}, y^{\prime}\right]^{\prime} \in \mathfrak{R}^{n+m}$.

\section{Problem Statement}

Consider the discrete-time linear system

$$
\begin{cases}x(t+1) & =A x(t)+B u(t) \\ y(t) & =C x(t)\end{cases}
$$

where $x(t) \in \mathfrak{R}^{n}, u(t) \in \mathfrak{R}^{m}, y(t) \in \mathfrak{R}^{p}$ are the state, the input and the measured output vectors, respectively, and $t \in \mathcal{N}$. Matrices $A, B$ and $C$ are real constant matrices of appropriate dimensions. Pairs $(A, B)$ and $(C, A)$ are assumed to be controllable and observable respectively.

The input vector $u$ is subject to amplitude limitations de£ned as follows:

- Amplitude constraints:

$$
\left|u_{(i)}(t)\right| \leq \rho_{a(i)}, \quad i=1, \ldots, m
$$

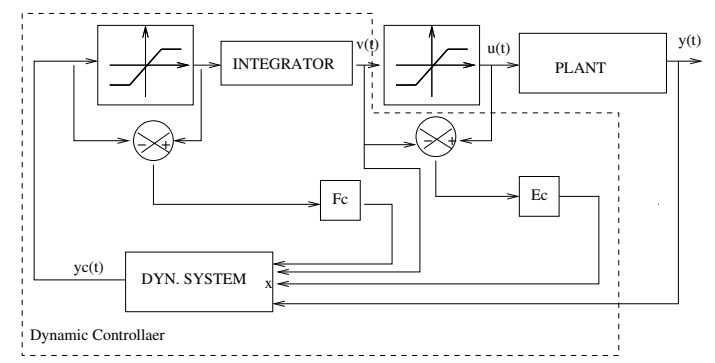

Fig. 1. closed-loop system

where $\rho_{a(i)}>0, i=1, \ldots, m$ denote the control amplitude bounds.

- Rate constraints:

$$
\left|\Delta u_{(i)}(t)\right|=\left|u_{(i)}(t)-u_{(i)}(t-1)\right| \leq \rho_{r(i)}, \quad i=1, \ldots, m
$$

where $\rho_{r(i)}>0, i=1, \ldots, m$ denote the rate control bounds.

We suppose that only the output $y(t)$ is available for measurement. Hence our aim is to compute a stabilizing dynamic compensator.

In order to cope with the rate constraints, in the sequel we consider a controller composed by an $n+m$ order dynamic compensator in cascade with $m$ input saturating integrators and two anti-windup loops, as follows:

$$
\begin{aligned}
v(t+1)= & I_{m} v(t)+\operatorname{sat}_{\rho_{r}}\left(y_{c}(t)\right) \\
x_{c}(t+1)= & A_{c} x_{c}(t)+B_{c}\left[y(t)^{\prime} \quad v(t)^{\prime}\right]^{\prime} \\
& +E_{c}\left(\operatorname{sat}_{\rho_{a}}(v(t))-v(t)\right) \\
& +F_{c}\left(\operatorname{sat}_{\rho_{r}}\left(y_{c}(t)\right)-y_{c}(t)\right) \\
y_{c}(t)= & C_{c} x_{c}(t)+D_{c}\left[y(t)^{\prime} v(t)^{\prime}\right]^{\prime}
\end{aligned}
$$

where $x_{c}(t) \in \mathfrak{R}^{n+m}$ is the dynamic compensator state, $y_{c}(t) \in \mathfrak{R}^{m}$ is the controller output, matrices $A_{c}, B_{c}, C_{c}, D_{c}$, $E_{c}$ and $F_{c}$ have appropriate dimensions. $E_{c}$ and $F_{c}$ are antiwindup gains.

As a consequence of the amplitude control bounds, the effective control signal applied to system (1) is a saturated one:

$$
u(t)=\operatorname{sat}_{\rho_{a}}(v(t))
$$

The resulting closed-loop system is nonlinear and can be written as

$$
\begin{aligned}
x(t+1)= & A x(t)+B \operatorname{sat}_{\rho_{a}}(v(t)) \\
v(t+1)= & I_{m} v(t)+\operatorname{sat}_{\rho_{r}}\left(y_{c}(t)\right) \\
x_{c}(t+1)= & A_{c} x_{c}(t)+B_{c}\left[y(t)^{\prime} v(t)^{\prime}\right]^{\prime} \\
& +E_{c}\left(\operatorname{sat}_{\rho_{a}}(v(t))-v(t)\right. \\
& +F_{c}\left(\operatorname{sat}_{\rho_{r}}\left(y_{c}(t)\right)-y_{c}(t)\right) \\
y_{c}(t)= & C_{c} x_{c}(t)+D_{c}\left[y(t)^{\prime} \quad v(t)^{\prime}\right]^{\prime}
\end{aligned}
$$

The whole closed loop system is depicted in fgure 1.

The main objective of the paper is therefore to compute matrices $A_{c}, B_{c}, C_{c}, D_{c}, E_{c}$ and $F_{c}$ in such a way that the domain of attraction of the closed-loop system is maximized under some performance constraints. 


\section{PRELIMINARIES}

For a given vector $\alpha \in \mathfrak{R}^{m}$ defne function $\psi_{\eta}(\alpha)$ as

$$
\psi_{\eta}(\alpha) \triangleq \alpha-\operatorname{sat}_{\eta}(\alpha) .
$$

Note that $\psi_{\eta}(\alpha)$ corresponds to a decentralized deadzone nonlinearity $\psi_{\eta}(\alpha)=\left[\begin{array}{lll}\left(\psi_{\eta}(\alpha)\right)_{(1)} & \ldots & \left(\psi_{\eta}(\alpha)\right)_{(m)}\end{array}\right]^{\prime}$, and

$$
\left(\psi_{\eta}(\alpha)\right)_{(i)}=\left\{\begin{array}{lll}
\alpha_{(i)}-\eta_{(i)} & \text { if } & \alpha_{(i)}>\eta_{(i)} \\
0 & \text { if } & -\eta_{(i)} \leq \alpha_{(i)} \leq \eta_{(i)} \\
\alpha_{(i)}+\eta_{(i)} & \text { if } & \alpha_{(i)}<-\eta_{(i)}
\end{array}\right.
$$

for all $i=1, \ldots, m$. Considering the generic nonlinearity $\psi_{\eta}(\alpha)$ and defning the set

$$
\begin{aligned}
S(\eta)= & \left\{(\alpha, \beta): \alpha, \beta \in \Re^{m},\left|\alpha_{(i)}-\beta_{(i)}\right| \leq \eta_{(i)},\right. \\
& i=1, \ldots, m\}
\end{aligned}
$$

the following lemma can be stated.

Lemma 1: [3] If $\alpha, \beta \in \mathfrak{N}^{m}$ are such that $(\alpha, \beta) \in S(\eta)$, then the nonlinearity $\psi_{\eta}(\alpha)$ satis£es the following inequality:

$$
\psi_{\eta}(\alpha)^{\prime} T\left(\psi_{\eta}(\alpha)-\beta\right) \leq 0
$$

for any diagonal positive de£nite matrix $T \in \mathfrak{R}^{m \times m}$.

Lemma 2: Consider the following system composed by $m$-integrators:

$$
\begin{aligned}
v(t+1) & =I_{m} v(t)+q(t) \\
u(t) & =\operatorname{sat}_{\rho_{a}}(v(t))
\end{aligned}
$$

If $\left|q(t)_{(i)}\right| \leq \rho_{r(i)}, i=1, \ldots, m$, it follows that

Proof:

$$
\left|\Delta u_{(i)}(t+1)\right|=\left|u_{(i)}(t+1)-u_{(i)}(t)\right| \leq \rho_{r(i)}
$$

Considering that the Lipschitz constant of the sat $(\cdot)$ function is equal to 1 , it follows directly that

$$
\begin{aligned}
\left|\Delta u_{(i)}(t+1)\right|= & \mid s a t_{\rho_{a(i)}}\left(v_{(i)}(t)+q_{(i)}(t)\right) \\
& -\operatorname{sat}_{\rho_{a(i)}}\left(v_{(i)}(t)\right) \mid \\
\leq & \left|q(t)_{(i)}\right| \leq \rho_{r(i)}
\end{aligned}
$$

$\diamond$

De£ne now the following vectors and matrices

$$
\begin{gathered}
\tilde{x}=\left[\begin{array}{l}
x \\
v
\end{array}\right] ; \xi=\left[\begin{array}{c}
\tilde{x} \\
x_{c}
\end{array}\right] \\
\mathbf{A}=\left[\begin{array}{cc}
A & B \\
0 & I_{m}
\end{array}\right] ; \mathbf{B}_{1}=\left[\begin{array}{c}
B \\
0
\end{array}\right] ; \mathbf{B}=\left[\begin{array}{c}
0 \\
I_{m}
\end{array}\right] \\
\mathbf{C}=\left[\begin{array}{cc}
C & 0 \\
0 & I_{m}
\end{array}\right] ; \mathbf{L}=\left[\begin{array}{ll}
0 & I_{m}
\end{array}\right] \\
\mathcal{A}=\left[\begin{array}{cc}
\mathbf{A}+\mathbf{B} D_{c} \mathbf{C} & \mathbf{B} C_{c} \\
B_{c} \mathbf{C} & A_{c}
\end{array}\right] ; \mathcal{B}_{1}=\left[\begin{array}{c}
\mathbf{B}_{1} \\
E_{c}
\end{array}\right] \\
\mathcal{B}=\left[\begin{array}{c}
\mathbf{B} \\
F_{c}
\end{array}\right] ; \mathcal{L}=\left[\begin{array}{ll}
\mathbf{L} & 0
\end{array}\right] ; \mathcal{K}=\left[\begin{array}{ll}
D_{c} \mathbf{C} & C_{c}
\end{array}\right]
\end{gathered}
$$

From the defnitions above, the closed-loop system can be re-written as

$$
\xi(t+1)=\mathcal{A} \xi(t)-\mathcal{B}_{1} \psi_{\rho_{a}}(\mathcal{L} \xi(t))-\mathcal{B} \psi_{\rho_{r}}(\mathcal{K} \xi(t))
$$

\section{Main Results}

Theorem 1: If there exist symmetric positive de£nite matrices $X, Y \in \mathfrak{R}^{(n+m) \times(n+m)}$, positive defnite diagonal matrices $S_{a}, S_{r} \in \mathfrak{R}^{m \times m}$, and matrices $\hat{A} \in$ $\mathfrak{R}^{(n+m) \times(n+m)}, \quad \hat{C} \in \mathfrak{R}^{m \times(n+m)}, \quad \hat{B} \in \mathfrak{R}^{(n+m) \times(p+m)}, \quad \hat{D} \in$ $\Re^{m \times(p+m)}, Z_{r 1}, Z_{r 2}, Z_{a 1}, Z_{a 2} \in \mathfrak{R}^{m \times(n+m)}, Q_{r}, Q_{a} \in \mathfrak{R}^{(n+m) \times m}$ such that the following inequalities are veri£ed ${ }^{1}$ :

$$
\begin{aligned}
& {\left[\begin{array}{cccccc}
X & \star & \star & \star & \star & \star \\
I_{n+m} & Y & \star & \star & \star & \star \\
Z_{r 1} & Z_{r 2} & 2 S_{r} & \star & \star & \star \\
Z_{a 1} & Z_{a 2} & 0 & 2 S_{a} & \star & \star \\
\mathbf{A} X+\mathbf{B} \hat{C} & \mathbf{A}+\mathbf{B} \hat{D} \mathbf{C} & \mathbf{B} S_{r} & \mathbf{B}_{1} S_{a} & X & \star \\
\hat{A} & Y \mathbf{A}+\hat{B} \mathbf{C} & Q_{r} & Q_{a} & I_{n+m} & Y
\end{array}\right]>0} \\
& {\left[\begin{array}{ccc}
X & \star & \star \\
I_{n+m} & Y & \star \\
\hat{C}_{(i)}-Z_{r 1(i)} & \hat{D}_{(i)} \mathbf{C}-Z_{r 2(i)} & \rho_{r(i)}^{2}
\end{array}\right] \geq 0}
\end{aligned}
$$

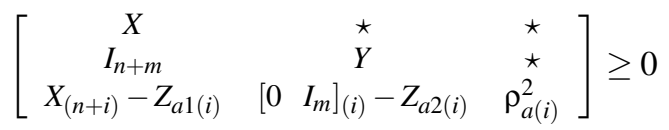

for all $i=1, \ldots, m$. Then the dynamic controller (4) with

$$
\begin{aligned}
F_{c}= & N^{-1}\left(Q_{r} S_{r}^{-1}-Y \mathbf{B}\right) \\
E_{c}= & N^{-1}\left(Q_{a} S_{a}^{-1}-Y \mathbf{B}_{1}\right) \\
D_{c}= & \hat{D} \\
C_{c}= & \left(\hat{C}-D_{c} \mathbf{C X}\right)\left(M^{\prime}\right)^{-1} \\
B_{c}= & N^{-1}\left(\hat{B}-Y \mathbf{B} D_{c}\right) \\
A_{c}= & N^{-1}\left[\hat{A}-\left(Y \mathbf{A} X+Y \mathbf{B} D_{c} \mathbf{C X}\right.\right. \\
& \left.\left.+N B_{c} \mathbf{C X}+Y \mathbf{B} C_{c} M^{\prime}\right)\right]\left(M^{\prime}\right)^{-1}
\end{aligned}
$$

where matrices $M$ and $N$ verify $N M^{\prime}=I_{n}-Y X$, guarantees that the region $\mathcal{E}(P) \triangleq\left\{\xi \in \mathfrak{R}^{2(n+m)}, \xi^{\prime} P \xi \leq 1\right\}$ with

$$
P=\left[\begin{array}{cc}
Y & N \\
N^{\prime} & \bullet
\end{array}\right] \quad P^{-1}=\left[\begin{array}{cc}
X & M \\
M^{\prime} & \bullet
\end{array}\right]
$$

is a region of asymptotic stability for the closed-loop system (10).

Proof:

Consider the closed loop system (10) and the candidate Lyapunov function $V(\xi(t))=\xi(t)^{\prime} P \xi(t), \quad P=P^{\prime}>0$. The variation of $V(\xi(t))$ along the trajectories of system (10) is given by

$$
\begin{aligned}
\Delta V(\xi(t))= & V(\xi(t+1))-V(\xi(t)) \\
= & -\xi(t)^{\prime} P \xi(t)+\xi(t)^{\prime} \mathcal{A}^{\prime} P \mathcal{A} \xi(t) \\
& -2 \xi(t)^{\prime} \mathcal{A}^{\prime} P \mathcal{B}_{1} \psi_{\rho_{a}}(\mathcal{L} \xi(t)) \\
& -2 \xi(t)^{\prime} \mathcal{A}^{\prime} P \mathcal{B} \psi_{\rho_{r}}(\mathcal{K} \xi(t)) \\
& +\psi_{\rho_{r}}(\mathcal{K} \xi(t))^{\prime} \mathcal{B}^{\prime} P \mathcal{B} \psi_{\rho_{r}}(\mathcal{K} \xi(t)) \\
& +\psi_{\rho_{a}}(\mathcal{L} \xi(t))^{\prime} \mathcal{B}_{1}^{\prime} P \mathcal{B}_{1} \psi_{\rho_{a}}(\mathcal{L} \xi(t)) \\
& +2 \psi_{\rho_{a}}(\mathcal{L} \xi(t))^{\prime} \mathcal{B}_{1}^{\prime} P \mathcal{B} \psi_{\rho_{r}}(\mathcal{K} \xi(t))
\end{aligned}
$$

Given matrices $G_{a}, G_{r} \in \mathfrak{R}^{m \times 2(n+m)}$, defne now the following sets

$$
\begin{aligned}
& \Xi\left(\rho_{a}\right) \triangleq\left\{\xi \in \mathfrak{R}^{2(n+m)} ;\left(\mathcal{L} \xi, G_{a} \xi\right) \in \mathcal{S}\left(\rho_{a}\right)\right\} \\
& \Xi\left(\rho_{r}\right) \triangleq\left\{\xi \in \mathfrak{R}^{2(n+m)} ;\left(\mathcal{K} \xi, G_{r} \xi\right) \in \mathcal{S}\left(\rho_{r}\right)\right\}
\end{aligned}
$$

${ }^{1} \star$ stands for symmetric blocks; • stands for an element that has no inquence on the development 
From Lemma 1, provided that $\xi(t) \in \Xi\left(\rho_{a}\right) \cap \Xi\left(\rho_{r}\right)$, it follows that

$$
\begin{aligned}
\Delta V(\xi(t)) \leq & \Delta V(\xi(t)) \\
& -2 \psi_{\rho_{r}}(\mathcal{K} \xi(t))^{\prime} T_{r}\left[\psi_{\rho_{r}}(\mathcal{K} \xi(t))-G_{r} \xi(t)\right] \\
& -2 \psi_{\rho_{a}}(\mathcal{L} \xi(t))^{\prime} T_{a}\left[\psi_{\rho_{a}}(\mathcal{L} \xi(t))-G_{a} \xi(t)\right]
\end{aligned}
$$

For ease of notation in the sequel we denote $\psi_{r}(t)=$ $\psi_{\rho_{r}}(\mathcal{K} \xi(t))$ and $\psi_{a}(t)=\psi_{\rho_{a}}(\mathcal{L} \xi(t))$. Therefore, expression (17) can be put in matrix form, as follows:

$$
\begin{aligned}
\Delta V(\xi(t)) \leq & -\theta(t)^{\prime}\left(\left[\begin{array}{ccc}
P & \star & \star \\
-T_{r} G_{r} & 2 T_{r} & \star \\
-T_{a} G_{a} & 0 & 2 T_{a}
\end{array}\right]\right. \\
& \left.-\left[\begin{array}{c}
-\mathcal{A}^{\prime} \\
\mathcal{B}^{\prime} \\
\mathcal{B}_{1}^{\prime}
\end{array}\right] P\left[\begin{array}{lll}
-\mathcal{A} & \mathcal{B} & \mathcal{B}_{1}
\end{array}\right]\right) \theta(t)
\end{aligned}
$$

where $\theta(t)=\left[\xi(t)^{\prime}, \psi_{r}(t)^{\prime}, \psi_{a}(t)^{\prime}\right]^{\prime}$.

From Schur's complement it follows that $\Delta V(\xi(t))<0$ if $\xi(t) \in \Xi\left(\rho_{a}\right) \cap \Xi\left(\rho_{r}\right)$ and

$$
\left[\begin{array}{cccc}
P & \star & \star & \star \\
-T_{r} G_{r} & 2 T_{r} & \star & \star \\
-T_{a} G_{a} & 0 & 2 T_{a} & \star \\
-\mathcal{A} & \mathcal{B} & \mathcal{B}_{1} & P^{-1}
\end{array}\right]>0
$$

Defne now a matrix $\Pi=\left[\begin{array}{cc}X & I_{n+m} \\ M^{\prime} & 0\end{array}\right]$ [12],[2]. Note now that, from (11), it follows that $I-X Y$ is non singular, which implies that is always possible to compute square and nonsingular matrices $N$ and $M$ verifying the equation $N M^{\prime}=$ $I-Y X$. This fact ensures that $\Pi$ is nonsingular.

Pre and post-multiplying (19) respectively by

$$
\left[\begin{array}{cccc}
-\Pi^{\prime} & 0 & 0 & 0 \\
0 & S_{r} & 0 & 0 \\
0 & 0 & S_{a} & 0 \\
0 & 0 & 0 & \Pi^{\prime} P
\end{array}\right] \text { and }\left[\begin{array}{cccc}
-\Pi & 0 & 0 & 0 \\
0 & S_{r} & 0 & 0 \\
0 & 0 & S_{a} & 0 \\
0 & 0 & 0 & P \Pi
\end{array}\right]
$$

with $S_{a}=T_{a}^{-1}$ and $S_{r}=T_{r}^{-1}$ one gets:

$$
\left[\begin{array}{cccc}
\Pi^{\prime} P \Pi & \star & \star & \star \\
G_{r} \Pi & 2 S_{r} & \star & \star \\
G_{a} \Pi & 0 & 2 S_{a} & \star \\
\Pi^{\prime} P \mathcal{A} \Pi & \Pi^{\prime} P \mathcal{B} S_{r} & \Pi^{\prime} P \mathcal{B}_{1} S_{a} & \Pi^{\prime} P \Pi
\end{array}\right]>0
$$

From the de£nition of $\Pi$, it follows that

$$
\begin{gathered}
\Pi^{\prime} P \Pi=\left[\begin{array}{cc}
X & I_{n+m} \\
I_{n+m} & Y
\end{array}\right] ; \Pi^{\prime} P \mathcal{B} S_{r}=\left[\begin{array}{c}
\mathbf{B} S_{r} \\
Y \mathbf{B} S_{r}+N F_{c} S_{r}
\end{array}\right] \\
\Pi^{\prime} P \mathcal{B}_{1} S_{a}=\left[\begin{array}{c}
\mathbf{B}_{1} S_{a} \\
Y \mathbf{B}_{1} S_{a}+N E_{c} S_{a}
\end{array}\right] \\
\Pi^{\prime} P \mathcal{A} \Pi=\left[\begin{array}{ll}
Q_{11} & Q_{12} \\
Q_{21} & Q_{22}
\end{array}\right]
\end{gathered}
$$

where

$$
\begin{aligned}
Q_{11}= & \left(\mathbf{A}+\mathbf{B} D_{c} \mathbf{C}\right) X+\mathbf{B} C_{c} M^{\prime} \\
Q_{12}= & \mathbf{A}+\mathbf{B} D_{c} \mathbf{C} \\
Q_{21}= & Y \mathbf{A} X+Y \mathbf{B} D_{c} \mathbf{C} X \\
& +N B_{c} \mathbf{C} X+Y \mathbf{B} C_{c} M^{\prime}+N A_{c} M^{\prime} \\
Q_{22}= & Y\left(\mathbf{A}+\mathbf{B} D_{c} \mathbf{C}\right)+N B_{c} \mathbf{C}
\end{aligned}
$$

Consider now the following change of variables $D_{c}=\hat{D}$, $C_{c} M^{\prime}+D_{c} \mathbf{C X}=\hat{C}, Y \mathbf{A} X+Y \mathbf{B} D_{c} \mathbf{C X}+N B_{c} \mathbf{C X}+Y \mathbf{B} C_{c} M^{\prime}+$ $N A_{c} M^{\prime}=\hat{A}, Y \mathbf{B} D_{c}+N B_{c}=\hat{B}, Y \mathbf{B} S_{r}+N F_{c} S_{r}=Q_{r}, Y \mathbf{B}_{1} S_{a}+$ $N E_{c} S_{a}=Q_{a}, G_{r} \Pi=\left[\begin{array}{ll}Z_{r 1} & Z_{r 2}\end{array}\right]$ and $G_{a} \Pi=\left[\begin{array}{ll}Z_{a 1} & Z_{a 2}\end{array}\right]$. Hence, since $\Pi, S_{r}$ and $S_{a}$ are nonsingular, it follows that if (11) is veri£ed, (19) holds with the matrices $A_{c}, B_{c}, C_{c}, D_{c}, E_{c}$ and $D_{c}$ defned as in (14).

On the other hand, considering that

$$
\begin{aligned}
& \mathcal{K} \Pi=\left[D_{c} \mathbf{C X}+C_{c} M^{\prime} \quad D_{c} \mathbf{C}\right]=\left[\begin{array}{ll}
\hat{C} & D_{c} \mathbf{C}
\end{array}\right] \\
& \left.\mathcal{L} \Pi=\left[\begin{array}{ll}
0 & I_{m}
\end{array}\right] \quad 0\right] \Pi=\left[\begin{array}{ll}
\tilde{X} & \tilde{I}
\end{array}\right]
\end{aligned}
$$

where $\tilde{X}$ and $\tilde{I}$ correspond respectively to the matrices composed by the last $m$ lines of matrices $X$ and $I_{n+m}$. Hence pre and post-multiplying inequalities (12) and (13) respectively by $\left[\begin{array}{cc}\left(\Pi^{-1}\right)^{\prime} & 0 \\ 0 & 1\end{array}\right]$ and its transpose, it is easy to see that (12) and (13) ensures respectively that $\mathcal{E}(P) \subset \Xi\left(\rho_{r}\right)$ and $\mathcal{E}(P) \subset \Xi\left(\rho_{a}\right)$.

Thus, if relation (11), (12) and (13) are satis£ed, one obtains $\Delta V(\xi(k))<0, \forall \xi(k) \in \mathcal{E}(P)$, which means that $\mathcal{E}(P)$ is a contractive region for system (10), i.e., $\forall \xi(0) \in \mathcal{E}(P)$, the corresponding trajectory converges asymptotically to the origin. $\diamond$

Theorem 2: If there exist symmetric positive de£nite matrices $X, Y \in \mathfrak{R}^{(n+m) \times(n+m)}$, positive de£nite diagonal matrices $S_{a}, S_{r} \in \mathfrak{R}^{m \times m}$, and matrices $\hat{A} \in \mathfrak{R}^{(n+m) \times(n+m)}$, $\hat{C} \in \mathfrak{R}^{m \times(n+m)}, \hat{B} \in \mathfrak{R}^{(n+m) \times(p+m)}, \hat{D} \in \mathfrak{R}^{m \times(n+m)}, Q_{r}, Q_{a} \in$ $\mathfrak{R}^{(n+m) \times m}$ such that the following inequalities are verifed

$$
\left[\begin{array}{cccccc}
X & \star & \star & \star & \star & \star \\
I_{n+m} & Y & \star & \star & \star & \star \\
\hat{C} & D_{c} \mathbf{C} & 2 S_{r} & \star & \star & \star \\
\tilde{X} & \tilde{I} & 0 & 2 S_{a} & \star & \star \\
\mathbf{A} X+\mathbf{B} \hat{C} & \mathbf{A}+\mathbf{B} \hat{D} \mathbf{C} & \mathbf{B} S_{r} & \mathbf{B}_{1} S_{a} & X & \star \\
\hat{A} & Y \mathbf{A}+\hat{B} \mathbf{C} & Q_{r} & Q_{a} & I_{n+m} & Y
\end{array}\right]>0
$$

where $\tilde{X}$ and $\tilde{I}$ correspond respectively to the matrices composed by the last $m$ lines of matrices $X$ and $I_{n+m}$; then the dynamic controller (4) with

$$
\begin{aligned}
F_{c}= & N^{-1}\left(Q_{r} S_{r}^{-1}-Y \mathbf{B}\right) \\
E_{c}= & N^{-1}\left(Q_{a} S_{a}^{-1}-Y \mathbf{B}_{1}\right) \\
D_{c}= & \hat{D} \\
C_{c}= & \left(\hat{C}-D_{c} \mathbf{C X}\right)\left(M^{\prime}\right)^{-1} \\
B_{c}= & N^{-1}\left(\hat{B}-Y \mathbf{B} D_{c}\right) \\
A_{c}= & N^{-1}\left(\hat{A}-\left(Y \mathbf{A} X+Y \mathbf{B} D_{c} \mathbf{C X}\right.\right. \\
& \left.\left.+N B_{c} \mathbf{C X}+Y \mathbf{B} C_{c} M^{\prime}\right)\right)\left(M^{\prime}\right)^{-1}
\end{aligned}
$$

where matrices $M$ and $N$ verify $N M^{\prime}=I_{n}-Y X$, guarantees the global asymptotic stability of the closed-loop system (10). 


\section{Proof:}

Consider $G_{r}=\mathcal{K}$ and $G_{a}=\mathcal{L}$. It follows that the sector conditions $\psi_{\rho_{r}}(\mathcal{K} \xi)^{\prime} T_{r}\left[\psi_{\rho_{r}}(\mathcal{K} \xi(t))-G_{r} \xi\right] \leq 0$ and $\psi_{\rho_{a}}(\mathcal{L} \xi)^{\prime} T_{a}\left[\psi_{\rho_{a}}(\mathcal{L} \xi(t))-G_{a} \xi\right] \leq 0$ are veri£ed for all $\xi \in$ $\mathfrak{R}^{2(n+m)}$. In this case, it is easy to see that (22) corresponds to (11) and the global asymptotic stability follows. $\diamond$

\section{Optimization PROBlems}

According to theorem 1, any feasible solution of the set of LMIs (11), (12) and (13) provides a stabilizing, and probably different, dynamic controller. Between all these solutions, one can be chosen to optimize a synthesis objective.

In this paper, the objective to be maximized will be the size of the domain of attraction on the closed-loop system, that is, the size of the projection of $\mathcal{E}(P)$ onto the states of the plant (i.e. $x$ ). This set is denoted as $\mathcal{E}_{x}(P)$ and is given by

$$
\begin{aligned}
\mathcal{E}_{x}(P) & =\left\{x \in \mathfrak{R}^{n} ; \exists v \in \mathfrak{R}^{m}, x_{c} \in \mathfrak{R}^{n+m} ;\left[x^{\prime} v^{\prime} x_{c}^{\prime}\right]^{\prime} \in \mathcal{E}(P)\right\} \\
& =\left\{x \in \mathfrak{R}^{n} ; x^{\prime} X_{11}^{-1} x \leq 1\right\}
\end{aligned}
$$

with $X_{11} \in \mathfrak{R}^{n \times n}$ is obtained from $X=\left[\begin{array}{cc}X_{11} & \star \\ X_{21} & X_{22}\end{array}\right]$.

Note that for any initial state $x(0) \in \mathfrak{E}_{x}(P)$, initial values of the states of the dynamic controller $v(0)$ and $x_{c}(0)$ can be found such that $\xi \in \mathcal{E}$, i.e. such that the asymptotically stability of the closed-loop system is ensured.

In order to maximize the size of $\mathcal{E}_{x}(P)$, the trace of $X_{11}$ is maximized. Note that the trace of $X_{11}$ equals to the sum of the semi-axis of the ellipsoid $\mathcal{E}_{x}(P)$. Consequently, the optimization problem to solve is

$$
\begin{aligned}
\max & \operatorname{trace}\left(X_{11}\right) \\
\text { s.t } & (11),(12),(13)
\end{aligned}
$$

It is worth noticing that performance requirements such as contraction rate, pole placement of the closed loop system or quadratic cost minimization can be added to the problem. In this case, the resulting optimization problem can be also written as an LMI optimization problem. Consider for instance, that the ellipsoid $\mathcal{E}(P)$ is required to be $\rho$-contractive, i.e if $\xi(t) \in \mathcal{E}(P)$ then $\xi(t+1) \in \mathcal{E}(P / \rho)$, where $\rho \in(0,1)$. This is equivalent to the following inequality:

$$
\Delta V(\xi(t))=\xi(t+1)^{\prime} P \xi(t+1)-\rho\left(\xi(t)^{\prime} P \xi(t)\right)<0
$$

It is easy to see that this condition is transformed in the solution of the following LMI:

$$
\left[\begin{array}{cccccc}
\rho X & \star & \star & \star & \star & \star \\
\rho I_{n+m} & \rho Y & \star & \star & \star & \star \\
Z_{r 1} & Z_{r 2} & 2 S_{r} & \star & \star & \star \\
Z_{a 1} & Z_{a 2} & 0 & 2 S_{a} & \star & \star \\
\mathbf{A} X+\mathbf{B} \hat{C} & \mathbf{A}+\mathbf{B} \hat{D} \mathbf{C} & \mathbf{B} S_{r} & \mathbf{B}_{1} S_{a} & X & \star \\
\hat{A} & Y \mathbf{A}+\hat{B} \mathbf{C} & Q_{r} & Q_{a} & I_{n+m} & Y
\end{array}\right]>0
$$

Thus, to satisfy a contraction rate, LMI (11) must be replaced by (24) in the optimization problem.

Remark 1: In [9], it was shown that, at least in some cases, the use of saturating control laws does not help in obtaining larger regions of stability. It is, however, very important to highlight that no constraints on the control rate, neither on the the performance, nor on the robustness, were taken into account in this analysis. In this case, although the optimal region of stability is obtained with a linear control law, the closed-loop poles associated to this solution can be very close to the imaginary axis, which implies a very slow behavior, as shown in [4]. In that paper, a clear trade-off between performance, effective saturation and the size of the region of stability of the closed-loop system is discussed.

\section{NUMERICAL EXAMPLE}

Consider the discrete-time linear system given by

$$
A=\left[\begin{array}{cc}
0.8 & 0.5 \\
-0.4 & 1.2
\end{array}\right], B=\left[\begin{array}{l}
0 \\
1
\end{array}\right], C=\left[\begin{array}{ll}
0 & 1
\end{array}\right]
$$

This system must be controlled with the following saturating limits

$$
|u(t)| \leq 1, \quad|\Delta u(t)| \leq 0.3
$$

The stabilizing dynamic controller has been computed solving the proposed optimization problem, i.e. maximizing the trace of $X_{11}$, with a contraction rate $\rho=0.8$. The obtained controller is given by

$$
\begin{aligned}
& A_{c}=\left[\begin{array}{ccc}
0.6769 & -0.0329 & 0.0262 \\
-0.8908 & 0.0433 & -0.0345 \\
0.3474 & -0.0174 & 0.0134
\end{array}\right] \\
& B_{c}=\left[\begin{array}{cc}
20.9653 & 8.8092 \\
68.3610 & 52.5307 \\
-14.1168 & -34.6764
\end{array}\right] \\
& C_{c}=\left[\begin{array}{lll}
0.0115 & -0.0006 & 0.0004
\end{array}\right] \\
& D_{c}=\left[\begin{array}{ll}
-0.3058 & -1.2195
\end{array}\right] \\
& E_{c}=\left[\begin{array}{c}
10.3491 \\
45.9644 \\
-82.6889
\end{array}\right] \quad F_{c}=\left[\begin{array}{c}
7.0142 \\
-29.9126 \\
-218.7285
\end{array}\right]
\end{aligned}
$$

The projection of the stability region $\mathcal{E}(P)$ onto the plant states is given by:

$$
\mathcal{E}_{x}(P)=\left\{x \in \mathfrak{R}^{2}: x^{\prime}\left[\begin{array}{cc}
0.1161 & -0.0430 \\
-0.0430 & 0.4729
\end{array}\right] x \leq 1\right\}
$$

In fgure 2 this contractive ellipsoid is shown as well as the trajectories of the controlled system for several initial states. For a given initial state, the initial controller states i.e $v(0)$ and $x_{c}(0)$, are chosen in such a way that $\xi(0)=$ $\left[x(0)^{\prime}, v(0)^{\prime}, x_{c}(0)^{\prime}\right]^{\prime}$ is in $\mathcal{E}(P)$.

In £gure 3 the evolution of the output system $y(t)$, control action $u(t)$ and increment of the control action $\Delta u(t)$ are depicted when the system starts from $x(0)=[-0.5,-1.45]^{\prime}$. Notice that the limit requirements in $u(t)$ and $\Delta u(t)$ are satis£ed thanks to the proposed saturating dynamic output feedback. In £gure 4, the evolution of the logarithm of the Lyapunov function $V(t)$ is shown. It can be seen that it is strictly decreasing and the contraction rate $\rho=0.8$ is veri£ed. 


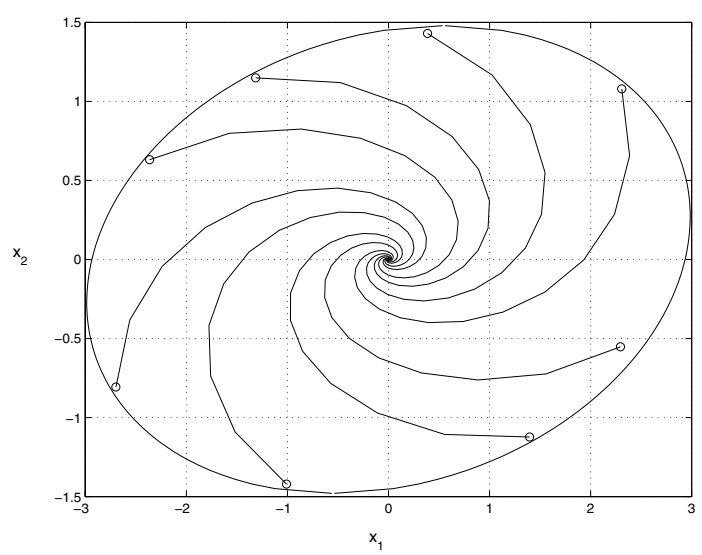

Fig. 2. Domain of attraction and state portrait of the controlled system.
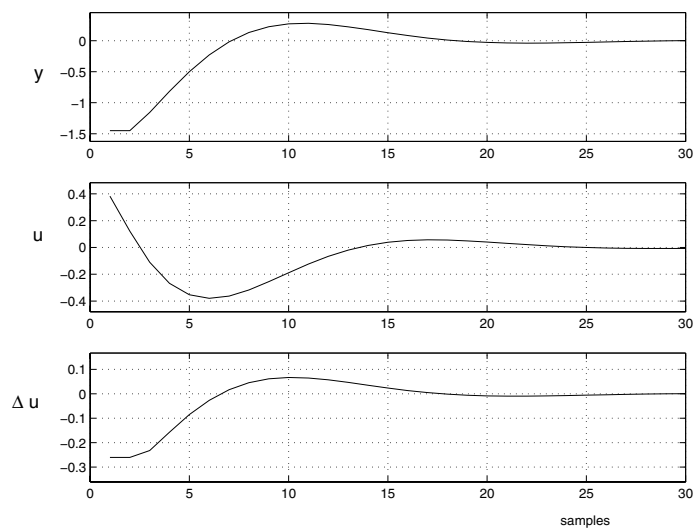

Fig. 3. Evolution of $y(t), u(t)$ and $\Delta u(t)$.

\section{CONCLUSIONS}

In this paper a technique for the design of stabilizing dynamic output feedback controllers for discrete-time linear systems with rate and amplitude constrained actuators has been proposed. This controller is composed by a classical linear dynamic compensator in cascade with an input saturating integrator system and two static antiwindup loops.

Theoretical conditions to ensure local and global stabilization of the closed-loop system, composed by the plant and the proposed controller, have been formulate in LMI form thanks to the use of a generalized sector condition . This allows to compute the controller matrices in order to maximize the size of the domain of attraction maintaining certain performance requirement from the solution of convex optimization problems. A numerical example has been provided to illustrate the application of the proposed method.

\section{REFERENCES}

[1] A. Bateman and Z. Lin. An analysis and design method for linear systems under nested saturation. System \& Control Letters, 48(1):4152, 2002.

[2] M. C. de Oliveira, J. C. Geromel, and J. Bernussou. Design of dynamic output feedback decentralized controllers via a separation procedure. Int. Journal of Control, 73(5):371-381, 2000.

[3] J. M. Gomes da Silva Jr. and S. Tarbouriech. Anti-windup design with guaranteed regions of stability: an lmi-based approach. IEEE Trans. on Automatic Control, 50(1):106-111, 2005.

[4] J. M. Gomes da Silva Jr., S. Tarbouriech, and G. Garcia. Local stabilization of linear systems under amplitude and rate saturating actuators. IEEE Trans. on Automatic Control, 48(5):842-847, 2003.

[5] G. Grimm, J. Hatfeld, I. Postlethwaite, A. Teel, M. Turner, and L. Zaccarian. Antiwindup for stable systems with input saturation: an lmi-based synthesis. IEEE Trans. on Automatic Control, 48(9):1500$1525,2003$.

[6] T. Hu and Z. Lin. Control systems with actuator saturation: analisys and design. Birkhauser, 2001.

[7] V. Kapila and K. Grigoriadis (Editors). Actuator Saturation Control. Marcel Dekker, Inc., 2002

[8] N. Kapoor, A. R. Teel, and P. Daoutidis. An anti-windup design for linear systems with input saturation. Automatica, 34(5):559-574, 1998.

[9] T. Kiyama and T. Iwasaki. On the use of multi-loop circle for saturating control synthesis. Systems \& Control Letters, 41:105-114, 2000.

[10] Z. Lin. Semi-global stabilization of linear systems with position and rate-limited actuators. System \& Control Letters, 30:1-11, 1997.

[11] T. Nguyen and F. Jabbari. Output feedback controllers for disturbance attenuation with actuator amplitude and rate saturation. Automatica, 36:1339-1346, 2000.

[12] C. Scherer, P. Gahinet, and M. Chilali. Multiobjective output-feedback control via lmi optimization. IEEE Trans. on Automatic Control, 42(7):896-911, 19971997.

[13] G. Shi, A. Saberi, and A. A. Stoorvogel. On $L_{p}$ performance with global internal stability for linear systems with actuators subject to amplitude and rate saturations. In Proceedings of the 19th American Control Conference (ACC'2000), pages 730-734, Chicago, USA, 2000.

[14] A. A. Stoorvogel and A. Saberi. Output regulation for linear systems to amplitude plus rate saturating actuators. Int. J. of Robust and Nonlinear Control, 9(10):631-657, 1999.

[15] S. Tarbouriech and G. Garcia (Editors). Control of uncertain systems with bounded inputs. Springer Verlag., 1997.

[16] S. Tarbouriech, C. Prieur, and J. M. Gomes da Silva Jr. Stability analysis and stabilization of systems presenting nested saturations. In Proc. of the 43rd IEEE Conference on Decision and Control, Paradise Island, Bahamas, 2004.

[17] F. Tyan and D. S. Bernstein. Dynamic output feedback compensation for linear systems with independent amplitude and rate saturation. Int. Journal of Control, 67(1):89-116, 1997.

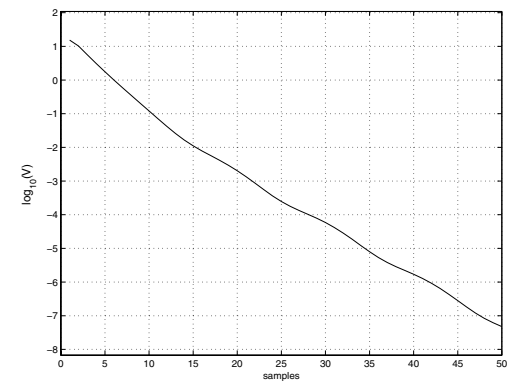

Fig. 4. Evolution of $\log _{10}(V(t))$. 